\title{
Perfil da demanda atendida em ambulatório de doenças profissionais e a presença de le- sões por esforços repetitivos
} Profile of occupational disease outpatients and the presence of repetitive strain injury

Ricardo J Reis ${ }^{a}$, Tarcísio MM Pinheiro ${ }^{a}$, Albert Navarro ${ }^{b}$ e Miguel Martin $\mathbf{M}^{\mathrm{b}}$

aAmbulatório de Doenças Profissionais do Hospital das Clínicas da Universidade Federal de Minas Gerais. Belo Horizonte, MG, Brasil. 'baboratori de Bioestadistica i Epidemiologia Facultat de Medicina, Universitat Autònoma de Barcelona. Barcelona, Spain 


\title{
Perfil da demanda atendida em ambulatório de doenças profissionais e a presença de lesões por esforços repetitivos* Profile of occupational disease outpatients and the presence of repetitive strain injury
}

\author{
Ricardo J Reis ${ }^{a}$, Tarcísio MM Pinheiroa ${ }^{a}$ Albert Navarro ${ }^{b}$ e Miguel Martin $\mathbf{M}^{\mathrm{b}}$ \\ aAmbulatório de Doenças Profissionais do Hospital das Clínicas da Universidade Federal de Minas \\ Gerais. Belo Horizonte, MG, Brasil. 'baboratori de Bioestadistica i Epidemiologia Facultat de Medici- \\ na, Universitat Autònoma de Barcelona. Barcelona, Spain
}

\section{Descritores}

Assistência ambulatorial" .

Transtornos traumáticos cumulativos, epidemiologia\#. Pacientes ambulatoriais" . Saúde ocupacional". Necessidades e demanda dos serviços de saúde. Ocupações.

\section{Keywords}

Ambulatory care ${ }^{\#}$. Cumulative trauma disorders, epidemiology $\mathrm{y}^{\#}$. Outpatient care ${ }^{\#}$. Occupational health".

\section{Resumo}

\section{Objetivo}

Caracterizar o perfil do paciente atendido em ambulatório de doenças profissionais de um hospital geral e, em especial, o portador da condição patológica mais freqüente: as lesões por esforços repetitivos (LER).

Métodos

Foram analisadas as 565 fichas-resumo de atendimento ambulatorial em saúde do trabalhador, em 1996, em função de variáveis sociodemográficas, de características do trabalho e de diagnóstico. Foram adotados os seguintes procedimentos: descrição univariável de todas as variáveis; análise bivariada, utilizando-se o teste do qui-quadrado $\left(\chi^{2}\right)$ para variáveis categóricas; comparação de médias ou amplitudes para variável categórica e outra quantitativa; análise multivariada por meio de modelos log-linear, com transformação logit, para detectar os grupos com maior proporção de LER.

\section{Resultados}

Houve predomínio no atendimento de pacientes do sexo feminino com menos de 40 anos, sendo que, nos casos de LER, esse predomínio mostrou-se mais acentuado. As ocupações agrupadas apareceram em ordem decrescente, na seqüência: administrativos, operários e prestadores de serviços, sendo 59,6\% pertencentes ao ramo de atividade econômica de serviços. As LER corresponderam a 56\% dos diagnósticos feitos nas primeiras consultas. Conclusões

Confirmou-se a tendência de mudança no perfil epidemiológico das demandas atendidas no ambulatório de doenças profissionais, no final da década de 80 , quando emergiram as LER. Deslocaram-se para uma minoria as antigas doenças profissionais decorrentes da exposição a riscos químicos e físicos, em função daquelas relacionadas às condições ergonômicas inadequadas.

\section{Abstract}

\section{Objective}

To assess the profile of outpatients seen at an occupational disease clinic of a general and repetitive strain injury (RSI) patients by using multivariate analyze.

\section{Methods}

The first 565 visit registries for the year 1996 were assessed regarding their social and demographics aspects, work characteristics, and diagnosis. All the variables 
were analyzed by univariate analysis method. Bivariate data were analyzed using Chi square test $\left(c^{2}\right)$ for categorical variables, comparison of means or ranges for quantitative and categorical variables. Multivariate data were analyzed using log-linear models with logit-transformation to identify the groups with a larger proportion of RSI.

\section{Results}

Women sought the occupational diseases clinic more frequently than men, and there was a predominance of RSI cases. The occupation distribution was in order of priority as following: office workers, factory workers, and service workers. Of them, 56.9\% were from the economic sector of services. RSI was seen in $56 \%$ of the patients diagnosed at their first visit.

\section{Conclusions}

The analysis confirms the trends seen in the 80 s regarding the epidemiological changes in occupational diseases, when RSI started to be identified and displaced to a secondary position the other common diseases (due to physical and chemical hazards) because of inadequacy of ergonomics.

\section{INTRODUÇÃO}

No Brasil, informações sobre morbimortalidade em saúde do trabalhador são geradas ainda de forma limitada, fragmentada e heterogênea. ${ }^{2}$ Levantamentos estatísticos oficiais não retratam o quadro real de como adoecem os trabalhadores. Há subnotificação importante no registro do número de acidentes do trabalho e de doenças profissionais no Brasil. ${ }^{2}$

No que se refere mais especificamente às doenças profissionais, a despeito de persistir a subnotificação, pode-se detectar que vem ocorrendo aumento significativo do seu registro. Tal fato é explicado basicamente pelo aumento da oferta de serviços de saúde do trabalhador na rede pública de saúde ${ }^{8}$ e pelo melhor acesso à informação do trabalhador e da população em geral, com referência ao tema saúde e trabalho. ${ }^{9}$

No presente trabalho objetivou-se caracterizar os pacientes atendidos no ambulatório de doenças profissionais de forma geral, com o fim de analisar a evolução da demanda e, por outro lado, avaliar a importância das lesões por esforços repetitivos (LER) e as características a elas associadas.

\section{MÉTODOS}

\section{Caracterização do local de estudo}

O estudo foi realizado em ambulatório de doenças profissionais (ADP) de um hospital geral da Universidade Federal de Minas Gerais, inserido na rede pública como centro de referência em saúde do trabalhador. É aberto à comunidade, sendo a sua clientela procedente de todo o Estado de Minas Gerais.

A maior demanda origina-se na região metropolitana de Belo Horizonte, capital do Estado, com três mi- lhões de habitantes, cuja população economicamente ativa encontra-se distribuída entre os seguintes ramos de atividade econômica: serviços (56\%), indústria $(29 \%)$ e comércio $(14 \%) .{ }^{3} \mathrm{O}$ paciente procura o ADP diante da suspeita de ser portador de doença profissional ou relacionada ao trabalho.

\section{Amostra}

A amostra estudada foi constituída por todas as fichas-resumo de atendimento ambulatorial em saúde do trabalhador de primeiras consultas, realizadas no ADP durante o ano de 1996. Nessas fichas são anotados: a identificação do trabalhador, do seu trabalho e da empresa, os diagnósticos (quais são, se suspeitos ou definidos) e sua relação com o trabalho (com nexo, sem nexo ou se é indefinido) e os encaminhamentos propedêuticos, terapêuticos e administrativos.

\section{Variáveis}

Para caracterização do paciente foram selecionadas as variavéis: sexo, idade, escolaridade, renda mensal (em salários-mínimos), ocupação (agrupada em prestadores de serviço, operários e administrativos), situação atual de trabalho, referência a outros serviços, relação ao mercado de trabalho, ramo de atividade econômica de atuação e diagnósticos, codificados segundo a Classificação Internacional de Doenças versão 9 - CID 9. ${ }^{6}$ Nas fichas-resumo constam, no máximo, cinco diagnósticos, a partir dos quais se classificou cada um nos grupos:

a) LER definidas: casos em que, sem dúvida, havia pelo menos um diagnóstico de doença osteomuscular dos membros superiores, com nexo definido com o trabalho.

b) LER indefinidas: casos com diagnóstico de doença osteomuscular dos membros superiores, nos 
quais o nexo com o trabalho não pôde ser firmado na primeira consulta.

c) não-LER: casos que indiscutivelmente não eram doenças osteomusculares.

A análise foi feita por meio dos pacotes estatísticos SPSS-WIN 6.0 e GLIM $4^{4}$ e compreendeu os seguintes procedimentos: (1) análise univariada de todas as variáveis; (2) análise bivariada, mediante teste de ANOVA de um fator para variáveis contínuas e Kruskal-Wallis, no caso de anormalidade. A associação entre as variáveis categóricas se determinou mediante o teste do $\chi^{2}$; e (3) análise multivariada foi feita por meio de modelo log-line$a r$, técnica adequada à descrição multivariada dos estudos transversais,,$^{1,10}$ descrevendo as associações entre as variáveis LER e o restante das variáveis, com a finalidade de caracterizar os grupos com maior probabilidade de apresentar LER.

Foram construídos os modelos 1 e 2, utilizando as variáveis LER, LER indefinidas e não-LER, e o modelo 3 com as variáveis LER e não-LER.

Em todos os casos, analisaram-se os logits correspondentes, cuja técnica mostrou-se, em outros estudos, muito útil para descrever grupos de risco e para a descrição multivariada, quando as variáveis são categóricas. ${ }^{9,11,12}$

Como todo modelo lineal generalizado, o ajuste pode-se efetuar com respeito a um valor médio, equivalente ao modelo linear de análise da variância na variável contínua, e tomar como referência uma célula qualquer da tabela multidimensional, gerada pelas variáveis categóricas. No presente caso, para cada modelo definiu-se uma célula de referência, sendo essa totalmente arbitrária, já que os resultados não se expressam em termos de odds ratio (OR), mas em prevalências de problemas nos grupos que definem as caselas da tabela multidimensional.

Com a finalidade de uma correta aplicação dos modelos logits, as demais variáveis consideradas também foram categorizadas, segundo os critérios de ida$d e$, recodificada nas seguintes categorias: $\mathrm{IDADE}=1$ (idade $\geq 40$ anos) e IDADE $=2$ (idade $<40$ anos) e situação atual de trabalho: $\mathrm{SIT}=1$ ou NA - não se aplica: refere-se a empregadores autônomos que não contribuem para o Instituto Nacional de Seguridade Social, aposentados e desempregados que não exercem atividades, donas-de-casa, estudantes e trabalhadores do mercado informal; $\mathrm{SIT}=2$ : trabalhadores ativos e SIT $=3$ : afastados do trabalho.

\section{RESULTADOS}

\section{O perfil da demanda do ADP}

Durante o ano de 1996, dentre as 565 primeiras consultas realizadas no ADP, $67,6 \%$ eram trabalhadores do sexo feminino; $60 \%$ dos pacientes atendidos estavam na faixa etária abaixo de 40 anos (mediana $=$ 37 ); $36,8 \%$ estudaram por pelo menos nove anos e a renda do trabalhador, calculada como a mediana de salários-mínimos recebidos, foi de três salários-mínimos por mês.

Pôde-se observar a presença de um grande número de ocupações (antes de serem agrupadas), sendo que as cinco mais freqüentes (auxiliar de escritório, faxineiro, digitador, cozinheiro e caixa) representaram aproximadamente $30 \%$ do total. Quando agrupadas (Tabela 1), distribuíram-se quase que por igual em prestadores de serviço, operários e administrativos.

Tabela 1 - Distribuição dos pacientes, segundo ocupação, em três grupos: ramo de atividade econômica, situação atual de trabalho, relação no mercado de trabalho e forma de referência - ADP, 1996.

\begin{tabular}{|c|c|c|}
\hline Variáveis & $N^{*}$ & $\%$ \\
\hline \multicolumn{3}{|l|}{ Ocupação** } \\
\hline Prestadores de serviços & 208 & 37,5 \\
\hline Operários & 176 & 31,7 \\
\hline Administrativos & 171 & 30,8 \\
\hline Total & 555 & \\
\hline \multicolumn{3}{|l|}{ Ramo de atividade econômica*** } \\
\hline Serviços & 310 & 59,6 \\
\hline Indústria & 153 & 29,4 \\
\hline Comércio & 57 & 11,0 \\
\hline \multirow{2}{*}{\multicolumn{3}{|c|}{ Situação atual de trabalho }} \\
\hline & & \\
\hline Ativo na mesma função & 258 & 46,1 \\
\hline Afastado & 186 & 33,2 \\
\hline NA & 90 & 16,1 \\
\hline Ativo com desvio de função & 26 & 4,6 \\
\hline Total & 560 & \\
\hline \multicolumn{3}{|l|}{ Relação no mercado de trabalho } \\
\hline Assalariado & 430 & 76,8 \\
\hline Desempregado & 71 & 12,7 \\
\hline Autônomo & 34 & 6,1 \\
\hline Aposentado & 15 & 2,7 \\
\hline Outros & 6 & 1,1 \\
\hline Empregador & 2 & 0,3 \\
\hline Mercado informal & 2 & 0,3 \\
\hline Total & 560 & \\
\hline \multicolumn{3}{|l|}{ Referência dos Serviços } \\
\hline Sem encaminhamento & 233 & 46,2 \\
\hline Sistema Único de Saúde & 133 & 26,4 \\
\hline Instituto Nacional Seguridade Social & 53 & 10,5 \\
\hline Sindicato & 26 & 5,2 \\
\hline Empresa & 19 & 3,8 \\
\hline Outros & 7 & 6,7 \\
\hline Serviço de saúde privado & 6 & 1,2 \\
\hline Total & 477 & \\
\hline
\end{tabular}

A mesma Tabela mostra que, no ramo da atividade econômica, a maioria dos trabalhadores se inseriu na área de serviços $(59,6 \%) ; 50,7 \%$ deles estavam em ativi- 
dade; $76,8 \%$ dos pacientes eram assalariados e pouco menos da metade deles $(46,2 \%)$ vieram sem encaminhamento, ou seja, sem referência de outros serviços.

Na Tabela 2 são apresentados os diagnósticos prevalentes, sendo que o grupo de doenças osteomusculares superou os $50 \%$ e, dentre essas, a síndrome do túnel do carpo constituiu-se na mais habitual. Dos dez diagnósticos mais freqüentes, nove eram doenças músculo-esqueléticas.

Tabela 2 - Distribuição dos diagnósticos em ordem descendente de freqüência - ADP, 1996.

\begin{tabular}{lrrr}
\hline Diagnóstico & $\mathrm{N}$ & $\%$ & $\begin{array}{r}\% \\
\text { Acumulado }\end{array}$ \\
\hline Síndrome do túnel do carpo & 117 & 11,1 & 11,1 \\
Dor em membro & 65 & 6,2 & 17,3 \\
Tenossinovite e sinovite & 58 & 5,5 & 22,8 \\
Tenossinovite estilóide & 56 & 5,3 & 28,2 \\
Síndrome do manguito rotador & 55 & 5,2 & 33,4 \\
Hipertensão essencial (primária) & 43 & 4,1 & 37,5 \\
Outra tenossinovite e sinovite & 38 & 3,6 & 41,1 \\
Tendinite supra-espinhoso & 38 & 3,6 & 44,7 \\
Cervicalgia & 31 & 2,9 & 47,7 \\
Mialgia & 20 & 1,9 & 49,6 \\
Outras afecções do ombro & 19 & 1,8 & 51,4 \\
Intoxicação por chumbo & 19 & 1,8 & 53,2 \\
Epicondilite lateral & 17 & 1,6 & 54,8 \\
Tendinite bicipital & 16 & 1,5 & 56,3 \\
Dermatite de contato não especificada & & \\
devida a outros agentes & 14 & 1,3 & 57,7 \\
Intoxicação por benzeno & 13 & 1,2 & 58,9 \\
Reumatismo não especificado & 12 & 1,1 & 60,0 \\
Dermatite de contato com químicos & 11 & 1,0 & 61,1 \\
Outros diagnósticos & 409 & 38,8 & 100,0 \\
\hline
\end{tabular}

\section{A presença da LER}

A soma total de diagnósticos resultou em 1.051, já que 330 pacientes receberam mais de um diagnóstico, 238 receberam apenas um e sete não receberam diagnóstico na primeira consulta. Os diagnósticos nãoLER representaram $44 \%$ do total, seguidos por LER definidas com $35 \%$ e por último LER indefinidas com $21 \%$. Ao se analisar e agregar os diagnósticos por trabalhador isoladamente, observa-se que $64 \%$ apresentavam um quadro suspeito ou confirmado de LER.

Na Tabela 3 mostram-se as distribuições sexo, idade, escolaridade, renda mensal, ocupação, ramo de atividade, situação atual de trabalho e encaminhamento, segundo diagnósticos causados ou não por LER. Como se pode observar, todas são significativas, excetuando-se a renda mensal e o encaminhamento.

Devido ao grande número de variáveis, a análise multivariada se efetuou ajustando-se, em primeiro lugar, dois modelos logits para relacionar as LER definidas, LER indefinidas e não-LER com as variáveis idade (recodificada), sexo e escolaridade, no primeiro modelo, e com os aspectos ocupacionais, no segundo.

Nos dois casos se construiu um modelo logit completo, ajustando-se convenientemente segundo cri-

Tabela 3 - Distribuição dos pacientes de acordo com o sexo, idade, escolaridade, renda mensal, ocupação, ramo de atividade econômica, situação atual de trabalho e referência, segundo o diagnóstico - ADP, 1996.

\begin{tabular}{|c|c|c|c|c|c|c|}
\hline Variáveis & & & & das & LEF & idas \\
\hline $\begin{array}{l}\text { Sexo }^{1} \\
\text { Masculino } \\
\text { Feminino }\end{array}$ & $\begin{array}{r}n \\
116 \\
86\end{array}$ & $\begin{array}{l}\% \\
57,4 \\
42,6\end{array}$ & $\begin{array}{r}n \\
28 \\
104\end{array}$ & $\begin{array}{l}\% \\
21,2 \\
78,8\end{array}$ & $\begin{array}{r}N \\
37 \\
187\end{array}$ & $\begin{array}{r}\% \\
16,5 \\
83,5\end{array}$ \\
\hline $\begin{array}{c}\text { Idade }(p=0,027) \\
\text { Menor de } 40 \text { anos } \\
40 \text { anos ou mais }\end{array}$ & $\begin{array}{r}109 \\
93\end{array}$ & $\begin{array}{l}54,0 \\
46,0\end{array}$ & $\begin{array}{l}77 \\
55\end{array}$ & $\begin{array}{l}58,3 \\
41,7\end{array}$ & $\begin{array}{r}149 \\
75\end{array}$ & $\begin{array}{l}66,5 \\
33,5\end{array}$ \\
\hline $\begin{array}{l}\text { Escolaridade }{ }^{*} \\
0 \text { a } 4 \text { anos } \\
5 \text { a } 8 \text { anos } \\
9 \text { anos ou mais }\end{array}$ & $\begin{array}{l}93 \\
56 \\
48\end{array}$ & $\begin{array}{l}47,2 \\
28,4 \\
24,4\end{array}$ & $\begin{array}{l}42 \\
36 \\
65\end{array}$ & $\begin{array}{l}32,3 \\
27,7 \\
40,0\end{array}$ & $\begin{array}{r}56 \\
65 \\
102\end{array}$ & $\begin{array}{l}25,1 \\
29,1 \\
45,7\end{array}$ \\
\hline $\begin{array}{l}\text { Renda mensal }(\mathrm{NS})^{*} \\
\text { Sem renda } \\
\text { Até } 3 \text { salários-mínimos } \\
\text { Mais de } 3 \text { salários-mínimos }\end{array}$ & $\begin{array}{l}20 \\
91 \\
57\end{array}$ & $\begin{array}{l}11,9 \\
54,2 \\
33,9\end{array}$ & $\begin{array}{l}15 \\
55 \\
46\end{array}$ & $\begin{array}{l}12,9 \\
47,4 \\
39,7\end{array}$ & $\begin{array}{l}21 \\
96 \\
83\end{array}$ & $\begin{array}{l}10,5 \\
48,0 \\
41,5\end{array}$ \\
\hline $\begin{array}{l}\text { Ocupação }{ }^{1 *} \\
\text { Operário } \\
\text { Administrativo } \\
\text { Prestadores de serviços }\end{array}$ & $\begin{array}{l}95 \\
28 \\
73\end{array}$ & $\begin{array}{l}48,5 \\
14,3 \\
37,2\end{array}$ & $\begin{array}{l}31 \\
44 \\
55\end{array}$ & $\begin{array}{l}23,8 \\
33,8 \\
42,3\end{array}$ & $\begin{array}{l}49 \\
97 \\
77\end{array}$ & $\begin{array}{l}22,0 \\
43,5 \\
34,5\end{array}$ \\
\hline $\begin{array}{l}\text { Ramo de atividade econômica }{ }^{1 *} \\
\text { Indústria } \\
\text { Comércio } \\
\text { Serviços }\end{array}$ & $\begin{array}{l}72 \\
15 \\
87\end{array}$ & $\begin{array}{r}41,4 \\
8,6 \\
50,0\end{array}$ & $\begin{array}{l}25 \\
11 \\
88\end{array}$ & $\begin{array}{r}20,2 \\
8,9 \\
71,0\end{array}$ & $\begin{array}{r}54 \\
31 \\
131\end{array}$ & $\begin{array}{l}25,0 \\
14,4 \\
60,6\end{array}$ \\
\hline $\begin{array}{l}\text { Situação atual de trabalho' }{ }^{1 *} \\
\text { Não se aplica } \\
\text { Ativo } \\
\text { Afastado }\end{array}$ & $\begin{array}{l}47 \\
98 \\
54\end{array}$ & $\begin{array}{l}23,6 \\
49,2 \\
27,1\end{array}$ & $\begin{array}{l}16 \\
76 \\
40\end{array}$ & $\begin{array}{l}12,1 \\
57,6 \\
30,3\end{array}$ & $\begin{array}{r}25 \\
107 \\
91\end{array}$ & $\begin{array}{l}11,2 \\
48,0 \\
40,8\end{array}$ \\
\hline $\begin{array}{l}\text { Referência (NS)* } \\
\text { Sim } \\
\text { Não }\end{array}$ & $\begin{array}{r}98 \\
100\end{array}$ & $\begin{array}{l}49,5 \\
50,5\end{array}$ & $\begin{array}{l}48 \\
81\end{array}$ & $\begin{array}{l}37,2 \\
62,8\end{array}$ & $\begin{array}{r}94 \\
128\end{array}$ & $\begin{array}{l}42,3 \\
57,7\end{array}$ \\
\hline
\end{tabular}

${ }^{1} \mathrm{p}<0,001$

* Refere-se apenas aos que informaram os dados 
tério de deviance não significativa e maior parcimônia.

O primeiro modelo foi estruturado sobre uma tabela de quatro dimensões LER *idade*sexo*escolaridade, o que define $3 * 2 * 2 * 3$ grupos observacionais diferentes.

No segundo modelo a tabela multidimensional analisada foi constituída por 81 grupos correspondentes às LER*ocupação*setor*situação no trabalho.

Na Tabela 4 podem-se observar as estimativas dos dois modelos ajustados em termos de proporção das três categorias das variáveis LER, em função dos fatores considerados.

No primeiro modelo log-linear obteve-se ajuste suficiente $\left(\mathrm{G}^{2}=18,4\right.$ g.l. $\left.=16, \mathrm{p}=0,302\right)$, com todas as interações de binárias entre as variáveis, o que permite derivar um modelo logit, no qual a variável LER, que tem três categorias, é função das outras três variáveis.

A situação que serve de referência no primeiro modelo é a do homem maior de 40 anos e com menos de cinco anos de estudo. Nessa situação basal, a proporção de LER indefinidas é de $13,7 \%$, enquanto que a de LER definidas é de $13,1 \%$. O efeito que se produz nessa situação e o fato de ser mulher supõem um aumento dessas categorias para $29,4 \%$ e $38,1 \%$, respectivamente. Esse efeito é superior ao que produz a mudança de grupo de idade ou de escolaridade.

A combinação dos três efeitos permite determinar a situação na qual a presença de LER definidas é mais provável: mulher, menor de 40 anos e com nível de escolaridade de mais de dez anos de estudo. Nessa situação, as LER definidas estão presentes em 59,1\% dos casos atendidos. Nas LER indefinidas estimou-se maior freqüência $(31,1 \%)$ no caso de mulheres, com o mesmo nível de escolaridade, porém maiores de 40 anos.

Definitivamente, retirando-se a variável idade, que se manteve no modelo por critério epidemiológico, apesar de ter significação estatística fraca $(p=0,08)$, o grupo mais acometido pelas LER, tanto nas categorias definidas como indefinidas, é constituído pelo grupo de mulheres com alta escolaridade. Nesse, $85,2 \%$ dos casos atendidos apresentam uma das duas categorias indicadas de LER.

No que se refere ao conjunto de variáveis relacionadas ao trabalho do paciente, o segundo modelo log-linear também obtém o ajuste suficiente com apenas interações binárias entre as quatro variáveis que formam a tabela multidimensional $\left(\mathrm{G}^{2}=62,2\right.$, g.1. $=48, \mathrm{p}=0,082$ ). O modelo logit derivado se estima com a situação de referência: operário pertencente à situação atual de trabalho NA, nas LER indefinidas em $12,7 \%$ dos casos, enquanto que nas LER definidas estão presentes em $22,2 \%$.

A ocupação é o fator que mais influencia a presença dessas duas categorias, sendo os administrativos os que apresentam uma proporção mais elevada de LER definidas $(53,6 \%)$, ao passo que a situação observada mais provável de apresentar LER é a con-

Tabela 4 - Estimativas dos três modelos ajustados em termos de proporção das três categorias da variável LER - ADP, 1996.

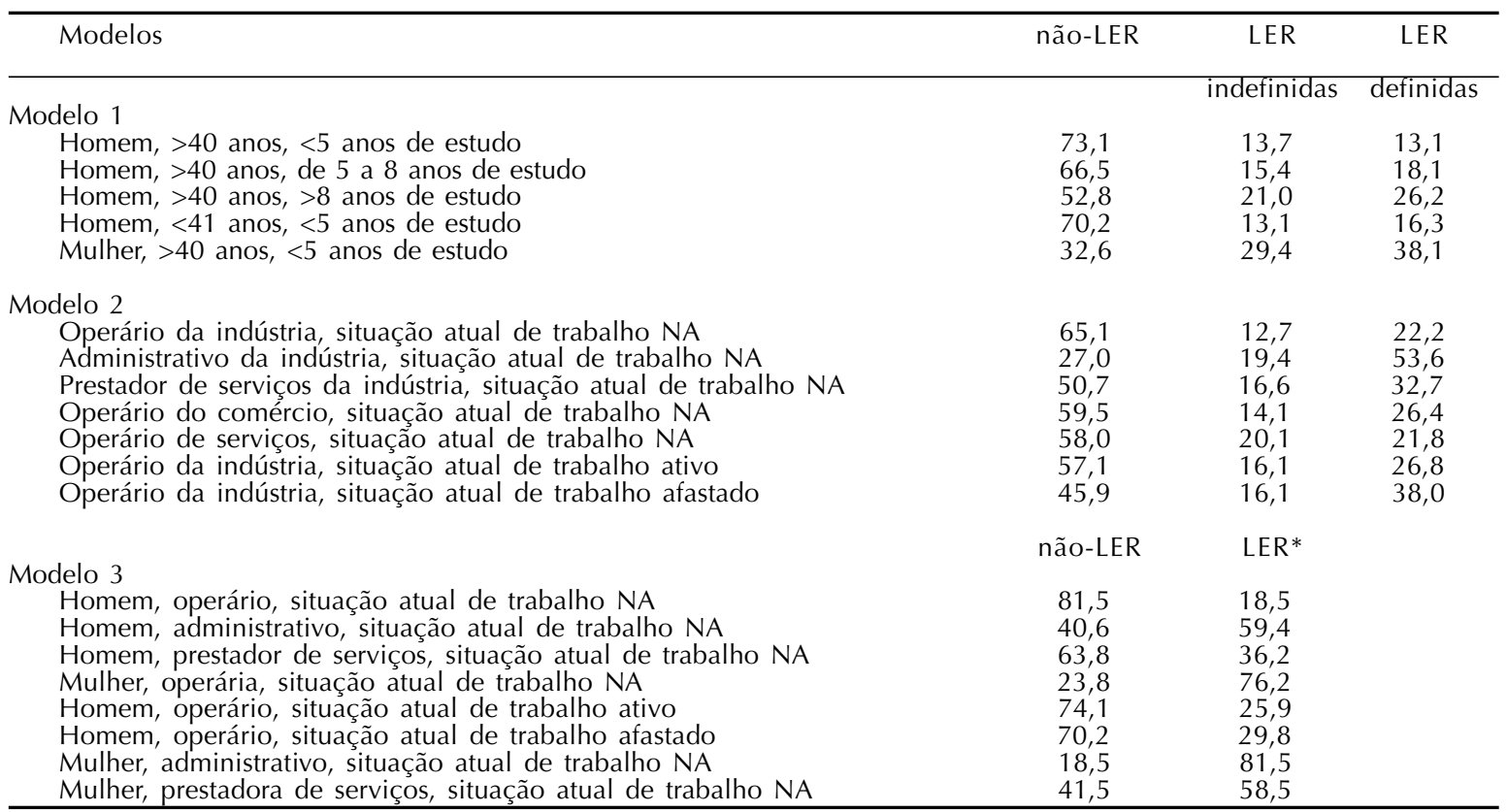

* Tanto indefinidas como definidas.

NA - Não se aplica 
figurada por administrativos do setor de comércio e afastados do trabalho, nos quais a categoria LER definidas se apresenta em 70,8\%.

Finalmente, a consideração conjunta das variáveis mais significativas obtidas nos dois modelos indica, num nível global, que o efeito da escolaridade e do ramo de atividade econômica desaparece ao se ajustar pela ocupação e pelo sexo, e que a idade não é significativa; portanto, a presença de LER ou nãoLER pode ser descrita satisfatoriamente a partir das variáveis sexo, ocupação e situação atual de trabalho.

Esse último modelo logit, Tabela 4, descritivo do fenômeno LER, apresentado em duas únicas categorias: não-LER e LER definidas ou indefinidas $\left(\mathrm{G}^{2}=16,0\right.$; g.l. $=14 ; \mathrm{p}=0,313$ ), prediz a segunda categoria em $89,2 \%$ dos casos atendidos, que respondem às seguintes características: mulher, afastada, administrativa.

\section{DISCUSSÃO}

\section{Demanda do ADP}

Até o final da década de 80 no ADP, "predominavam os trabalhadores do sexo masculino, com um crescimento gradual de trabalhadoras, na faixa de 20 a 40 anos". ${ }^{3}$ Observa-se que, atualmente, o número de pacientes do sexo feminino é o dobro daquele do sexo masculino, mantendo essa proporção estável, quando se faz a estratificação por idade. Ao se agregar por categoria de diagnósticos, observa-se que a razão homem/mulher é 1,3 no grupo não-LER, enquanto que nos grupos de LER há uma inversão com predomínio das mulheres, na proporção de três para cada homem. Há uma associação clara entre o aumento do número de mulheres atendidas no ADP em 1996 e o grande número de portadores de LER.

A maioria dos pacientes atendidos tem menos de 40 anos de idade. Isso revela um problema de grandes proporções, uma vez que a definição de um diagnóstico de doença profissional implica, geralmente, afastamento do trabalho numa faixa etária plenamente produtiva, fazendo com que saiam do mercado trabalhadores que poderiam estar contribuindo para o desenvolvimento da economia e que passam, pelo contrário, a depender de benefícios da Previdência Social.

No que se refere à escolaridade, $36,8 \%$ estudou pelo menos nove anos, ou seja, tem o primeiro grau, o que teoricamente facilitaria a reinserção no mercado de trabalho, em casos de reabilitação profissional.

Com referência à ocupação, existe uma distribuição homogênea quando se consideram os três gru- pos (operários, administrativos e prestadores de serviços) em conjunto, mas percebe-se claramente o predomínio absoluto dos operários nos grupos não-LER e dos administrativos e prestadores de serviços nos grupos LER.

Quanto à atividade das empresas, a maioria dos pacientes do ADP provém do ramo de atividade econômica de serviços, seguido por indústria e finalmente pelo comércio.

Ao se tratar a situação atual de trabalho, a maioria encontra-se em atividade, sugerindo que o ADP é realmente o primeiro serviço de saúde a ser procurado, o que é corroborado pelo fato de que quase a metade comparece à primeira consulta sem referência. A principal explicação para tal fato seria talvez a facilidade para se marcar consulta no ADP, feita por meio de procura direta, apesar dele ser considerado Centro de Referência em Saúde do Trabalhador no Estado.

\section{A presença da LER}

Ao se considerar apenas o grupo de LER, nota-se que a razão mulher/homem eleva-se para 4,47:1, ao contrário da amostra geral, que, apesar do predomínio do sexo feminino, é de aproximadamente a metade. Seriam as LER mais freqüentes em mulheres? Essa tem sido uma questão bastante debatida. Não está claro se a diferença de distribuição quanto ao sexo em algumas desordens osteomusculares dos membros superiores, constatadas em alguns estudos, é devida a diferenças fisiológicas (fibras musculares, estatura, maior freqüência no relato de dor) ou a diferenças na exposição (postos de trabalho inadequados, emprego em trabalhos manuais intensivos). Entretanto, há consenso nas diferenças existentes entre os homens e as mulheres com relação à inserção no mercado de trabalho. Esse fato repercute no modo como trabalhadores adoecem. Apesar de toda controvérsia, é fato no presente estudo, e confirmando tendência dos últimos anos, que há o predomínio das mulheres portadoras de LER em relação aos homens. Em estudo realizado no Centro de Reabilitação Profissional de Belo Horizonte, ${ }^{9}$ demonstrou-se que "os incapacitados por lesões por esforços repetitivos no programa de reabilitação (1995) eram na maioria mulheres."

Um diagnóstico de LER definidas pôde ser feito com maior frequiência em mulheres abaixo dos 40 anos e com escolaridade acima de nove anos. Por outro lado, um diagnóstico de LER indefinidas é mais freqüente também nas mulheres, mas naquelas acima de 40 anos e com o mesmo nível de escolaridade. Poder-se-ia inferir desses dados que após 40 anos de idade as doenças degenerativas estão mais 
presentes, fazendo com que a faixa de diagnóstico diferencial se amplie, necessitando-se definir o diagnóstico numa etapa além da primeira consulta. A maior prevalência dos administrativos nos casos de LER definidas pode ser explicada em se tratando de uma cidade em que se predomine o ramo de atividades de serviços, no qual se espera prevaleçam as atividades administrativas.

\section{REFERÊNCIAS}

1. Agresti A. Categorical data analysis. New York: John Willey and Sons, 1990.

2. Alves S, Luchesi G. Acidentes do trabalho e doenças profissionais no Brasil: a precariedade de informações. Informe Epidemiológico do SUS 1992;1(3):5-19.

3. Dias EC. Um pouco de história. Bol ADP 1993;1(1):1-3

4. Glim 4: the statistical system for generalized linear interactive modelling [computer program]. Oxford. (UK): Oxford University Press; 1993.

5. Ministério do Trabalho. Classificação Nacional de Atividades Econômicas: Portaria $\mathrm{n}^{\circ}$ 1. Brasília (DF); 1995.

6. Ministério do Trabalho. Secretaria de Políticas de Emprego e Salários. Classificação brasileira de ocupações. Brasília (DF); 1994.

7. Organização Mundial de Saúde. Revisão da Classificação Internacional de Doenças da (CID 9a. rev.) Baseada nas recomendações da $9^{a}$ Conferência de Revisão, 1975 e adotada pela 20ª Assembléia Mundial de Saúde. Porto Alegre: Sagra; 1992.

8. Rego MAV. Acidentes e doenças do trabalho no Estado da Bahia. 1970 a 1992. Informe Epidemiológico do SUS 1993;2(6):25-39.

9. Sampaio RF. Cuando los accidentes de trabajo no matan: una aproximación epidemiológica de las incapaciades permanentes para la profesión habitual [tesis]. Barcelona: Facultat de Medicina. Universitat Autònoma de Barcelona;1997.

10. Sampaio RF, Martin-Mateo M, Artazcoz L, Moncada SL. Acidentes de trabalho em Barcelona (Espanha), no período de 1992-1993. Rev Saúde Pública 1998;32:345-51.

11. Sanchez-Perez HJ, Ochoa-Diaz H, Garcia-Gil MM Martin-Mateo, M. Bienestar social y servicios de salud en la Región Fraylesca de Chiapas: el uso de servicios de atención prenatal. Salud Pública Mex 1997;39:530-8.

12. Sanchez-Perez HJ, Ochoa-Diaz H, Navarro-Gine, A, Martin-Mateo, M. Atención del parto en Chiapas, México: ¿Quién y dónde lo atiende? Salud Publica Mex 1998;40:494-502. 